\title{
Una Herramienta para el Análisis de la Colaboración Diseñada con UWE
}

\section{A Collaboration Analysis Tool Designed with UWE}

\author{
Ofelia Uicab Aldana, Juan P. Ucán Pech, Raúl A. Aguilar Vera \\ Facultad de Matemáticas \\ Universidad Autónoma de Yucatán \\ Mérida, Yucatán, México \\ A13016861@alumnos.uady.mx, \{juan.ucan, avera\}@correo.uady.mx
}

\begin{abstract}
Resumen - UML Web Engineering (UWE) es uno de los métodos en Ingeniería Web para el diseño de aplicaciones que se desarrollan para la World Wide Web; utiliza la simbología estándar del Lenguaje de Modelado Unificado, así como simbología propia para desarrollo orientado a la Web. En este trabajo se presenta el diseño de una aplicación Web desarrollada como herramienta de apoyo para analizar la interacción (HAI) de estudiantes en su proceso de aprendizaje, donde el modelado básico se realiza mediante UWE. Dicha aplicación recupera el registro de publicaciones que realizan los estudiantes en el muro grupal de Facebook y aplica un modelo diseñado para el análisis de la colaboración con el fin de identificar las habilidades colaborativas de cada uno de los estudiantes.
\end{abstract}

Abstract - UML Web Engineering (UWE) is one of the methods in Web Engineering for the design of applications that are developed for the World Wide Web; Uses the standard symbology of the Unified Modeling Language, as well as its own for Web-oriented development. This paper presents the design of a Web application developed as a support tool to analyze the interaction - called HAI by its acronym in Spanish-of students in their learning process, where the basic modeling was performed using UWE. This application retrieves the record of publications that the students make in the Facebook Wall and applies a model designed for the analysis of the collaboration in order to identify the collaborative abilities of each of the students.

Palabras clave - Aprendizaje Colaborativo Asistido por Computadora, Indicadores de Colaboración, Ingeniería Web, UWE.

Index Terms - Collaboration Indicators, Computer Supported Collaborative Learning, UWE, Web Engineering.

\section{INTRODUCCIÓN}

En este artículo se describe el diseño de una aplicación Web desarrollada como herramienta de apoyo para analizar la colaboración en escenarios de aprendizaje; el proceso de modelado se realiza mediante UWE (UML Web Engineering), uno de los métodos en Ingeniería Web para el diseño de aplicaciones que se desarrollan para la WWW (World Wide $W e b)$ con base en la simbología estándar UML y un conjunto de símbolos propios para representar el desarrollo web. La Herramienta para el Análisis de la Interacción (HAI) recupera el registro de publicaciones hechas por los estudiantes - luego de realizar una tarea colaborativa- en el muro grupal de Facebook, y les aplica un mecanismo de modelado para obtener indicadores que representan la colaboración, o dicho de otra forma, las habilidades colaborativas de los estudiantes y de los grupos de trabajo.
En la siguiente sección se presenta en breve el estado de arte en el que se sustenta el trabajo, en la tercera sección se describe el Framework para el Análisis de la Colaboración diseñado por los autores; la cuarta sección presenta el diseño de la arquitectura software, como cada uno de los modelos para la HAI desarrollados con base en UWE; las prestaciones de la HAI son descritas en la sección seis, y finalmente, en la última sección se describen las conclusiones y trabajos en curso.

\section{ESTADO DEL ARTE}

El concepto Groupware ha sido concebido como sistemas basados en computadoras que apoyan a grupos de personas que trabajan en una tarea u objetivo en común y que proveen una interfaz en un ambiente compartido [1]; los sistemas Groupware son desarrollados bajo el enfoque del Trabajo Cooperativo asistido por Computadora (CSCW por sus siglas en inglés), que es un campo multidisciplinario de donde deriva el paradigma del Aprendizaje Colaborativo Asistido por Computadora (CSCL por sus siglas en inglés), la cual representa una línea de investigación emergente en las ciencias del aprendizaje que se ocupa del estudio de cómo las personas pueden aprender juntos con la ayuda de computadoras [2]. En [3] se demostró la efectividad del aprendizaje colaborativo frente a un aprendizaje individual y se fueron identificando variables que han sido ampliamente estudiadas [4]. En este ámbito, la colaboración es definida como "el mutuo compromiso de los participantes en un esfuerzo coordinado por resolver problemas juntos" [5].

La implementación de escenarios para promover el aprendizaje colaborativo requiere una planeación, no es algo espontáneo pues parte de la responsabilidad del aprendizaje recae sobre los estudiantes, por lo que para que sea implementado exitosamente, se deben considerar varios factores, entre estos están el rol del profesor como facilitador pues él puede observar, corregir y trabajar con sus estudiantes para mejorar su rendimiento, teniendo como responsabilidad, orientar a los estudiantes en el objetivo y propósito de la técnica colaborativa así como deberá tomar decisiones sobre el tamaño del grupo, duración y operación de los grupos de aprendizaje. Además de diseñar y asignar tareas de aprendizaje para asegurar una activa y constructiva participación y evaluar el aprendizaje.

El desarrollo de aplicaciones para la World Wide Web, requiere aplicar Ingeniería Web [6], una disciplina enfocada al proceso de desarrollo de aplicaciones de alta calidad. Para el desarrollo de los modelos conceptuales de dichas aplicaciones, 
existen varias alternativas a seleccionar: OOHDM (ObjectOriented Hypermedia Design Model) [7], WebML (Web Modeling Language) [8], UWE [9], ésta última se basa en la notación estándar del UML (Unified Modeling Language) y adiciona elementos propios del desarrollo Web.

UWE es una metodología de la Ingeniería Web que contempla un conjunto de modelos con los cuales es posible diseñar la aplicación Web; dichos modelos son los siguientes:

- Modelo de requerimientos. Plasma los requisitos funcionales de la aplicación Web mediante un modelo de casos de uso.

- Modelo de contenido. Define, mediante un diagrama de clases, los conceptos involucrados en la aplicación.

- Modelo de navegación. Presenta la navegación dentro de la aplicación y un conjunto de estructuras como son índices, menús y consultas para.

- Modelo de presentación. Presenta las vistas de las interfaces de usuario de forma abstracta.

- Modelo de proceso. Representa el aspecto que tienen las actividades que se conectan con cada clase de proceso.

\section{MECANISMO PARA EL ANÁLISIS DE LA COLABORACIÓN}

El modelado de un grupo proporciona representaciones funcionales que nos ayudan a entender y explicar la interacción entre los integrantes de un grupo, pero también permite obtener información que nos permite retroalimentar el diseño instruccional utilizado [10].

En este proyecto, el modelado de la interacción se realizó utilizando un conjunto de claves y constructos diseñados para identificar la intención del habla, denominados Oraciones de Apertura [11] con las cuales es posible estructurar el diálogo mantenido por los estudiantes durante su proceso de aprendizaje, y con ello obtener, posteriormente, un conjunto de indicadores de la interacción vinculados con las cuatro habilidades colaborativas consideradas en nuestro trabajo, y propuestas en [12]. La interacción de los estudiantes es mediada a través del uso de prestaciones de la red social Facebook, en particular, el muro grupal.

En [13] los indicadores utilizados en el análisis de la interacción indican algo relacionado con la calidad de la actividad individual (p.e. variables que el/ella cambian, orden o significancia de las acciones, etc.), el modo o calidad de la colaboración (p.e. división de labores, intensidad de la colaboración, medida de participación, etc.), el proceso o la calidad del producto colaborativo. Esas variables tienen que ser interpretadas tomando en consideración la actividad de aprendizaje, el perfil de los participantes, el contexto de la interacción, etc. La Figura 1, presenta una taxonomía de subhabilidades ligadas a atributos y oraciones de apertura para la habilidad de comunicación.

Con este mecanismo de obtención de datos, para cada integrante del grupo se mantiene un par de valores relacionados con el uso (booleano) y frecuencia (entero) para cada uno de los 35 atributos considerados con las habilidades colaborativas: Comunicación (C: 10 atributos), Mantenimiento de la Confianza (M: 5 atributos), Dirección o Liderazgo (D: 8 atributos) y Gestión de conflictos (G: 12 atributos), y sobre los cuales se definen los indicadores.

En este trabajo se ha utilizado una adaptación del Modelo de Indicadores propuesto en [14], y se han generado cuatro conjuntos de indicadores, similares a los propuestos en [10]. Los cuatro conjuntos de indicadores son obtenidos para representar:

\begin{tabular}{|c|c|c|}
\hline \multicolumn{3}{|c|}{ Comunicación } \\
\hline Sub-habilidad & Atributo & Oración de apertura \\
\hline \multirow{3}{*}{ Remisión } & Apertura & C01 Pienso... \\
\hline & Completitud & $\begin{array}{l}\text { C02 Necesitas más } \\
\text { información... }\end{array}$ \\
\hline & $\begin{array}{l}\text { Compartir información } \\
\text { Ofrecer realimentación }\end{array}$ & $\begin{array}{l}\text { C03 Te paso... } \\
\text { C04 iEntiendes }\end{array}$ \\
\hline \multirow[t]{3}{*}{ Recepción } & Paráfrasis & C05 Entonces piensas que... \\
\hline & Percepción & $\begin{array}{l}\text { C06 Lo que entiendo que tú } \\
\text { crees, es que... }\end{array}$ \\
\hline & Negociación & C07 Creo que deseas.. \\
\hline \multirow[t]{3}{*}{ Reconocimiento } & Positivo & $\mathrm{C} 08 \mathrm{Si}$ \\
\hline & Negativo & C09 No \\
\hline & Agradecimiento & C10 Gracias \\
\hline
\end{tabular}

Fig.1. Oraciones de Apertura vinculadas al conjunto de habilidades-atributos para la Comunicación.

(1) Habilidades colaborativas — inferidas por su uso en una sesión de trabajo- que los estudiantes poseen.

(2) Habilidades colaborativas —inferidas por su uso en una sesión de trabajo- que el grupo posee.

(3) La participación de cada uno de los miembros y del grupo en una sesión de trabajo está constituido por $\mathrm{P}$ y PG respectivamente.

(4) Habilidades colaborativas usadas por los estudiantes en una sesión de trabajo.

\section{DiSEÑo DE LA HERRAMIENTA PARA EL ANÁlisis DE LA INTERACCIÓN (HAI).}

En esta sección se presenta el diseño de una aplicación Web desarrollada como herramienta de apoyo para analizar la colaboración, donde el modelado básico se realiza mediante UWE [9], uno de los métodos en Ingeniería Web para el diseño de aplicaciones que se desarrollan para la WWW. Dicha aplicación recupera el registro de publicaciones hechas por los estudiantes en el muro grupal de Facebook, es decir, la interacción registrada al realizar una tarea colaborativa, y con base en los mismos, se ejecuta el mecanismo de modelado implementado con la aplicación; con el cual se obtienen los conjuntos de indicadores, los cuales permiten al tutor humano analizar la colaboración de los estudiantes.

\section{A. Interconectividad con Facebook}

Para lograr el objetivo de obtener una aplicación Web como herramienta de apoyo para el análisis de la colaboración de grupos, se ha aprovechado la base sólida para la construcción de aplicaciones por terceros para Facebook a través del uso de uno de los SDK (Software Development Kit) para acceder a la API de Facebook, así como las herramientas de desarrollo que ofrece, como documentación y guía, así como el código disponible en el sitio de desarrolladores.

En la Figura 2, se muestra el diagrama del funcionamiento y módulos de la aplicación, cuando un grupo de estudiantes realiza una actividad grupal teniendo como medio de comunicación el muro grupal de Facebook, y empleando oraciones de apertura (OA) en sus publicaciones, el servidor de Facebook mantiene el registro de dicha información en su Base de Datos; luego la HAI haciendo uso del SDK y de la API de Facebook obtiene el registro de las publicaciones en formato JSON (Java Script Object Notation) el cual es procesado para generar el conjunto de indicadores aplicando un modelo de colaboración las cuales son representados en gráficas sencillas, así la aplicación nos permitirá:

- Saber de cada uno de los estudiantes y de los grupos, el nivel de cada una de las habilidades de colaboración que posee y de su habilidad colaborativa en general.

- Saber de cada uno de los estudiantes y de los grupos, la participación que tuvieron. 
- Realizar una comparación entre integrantes de equipo, y equipos para obtener el que posee mayor habilidad colaborativa y el que más participó, así como los que menos actividad reflejaron.

- Saber de cada uno de los estudiantes y de los grupos, cuál fue la habilidad más usada y menos usada.

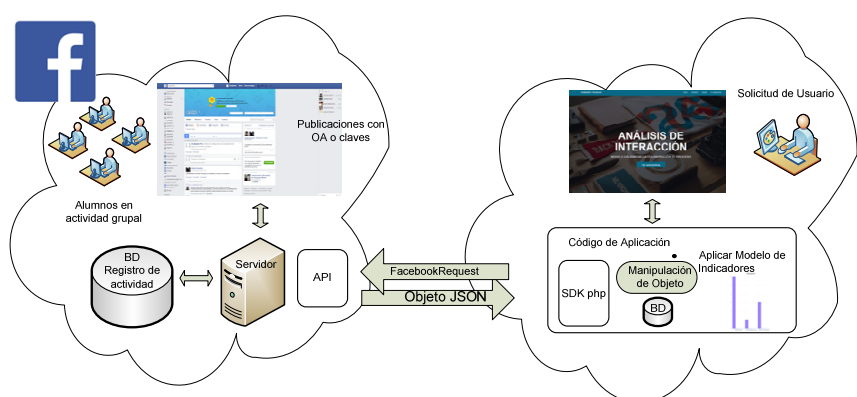

Fig.2. Diagrama del funcionamiento y módulos de la arquitectura propuesta de la aplicación.

Por lo tanto, los equipos con mayor nivel colaborativo se espera que sean quienes hayan tenido un mayor aprendizaje además de haber mejorado sus habilidades colaborativas. Con estos resultados el profesor podría optar por alguna medida para aquellos estudiantes que reflejaron baja participación y fueron pobres en la habilidad analizadas, pues el objetivo final es identificar quién de los integrantes necesita reforzamiento, ayuda o algún tipo de apoyo.

Los usuarios de la aplicación pueden ser el profesor, el alumno, el investigador y todo aquel interesado en evaluar la colaboración en grupos debido a que la aplicación sólo requiere tener una sesión en Facebook activa al momento de correr la aplicación, además que los grupos creados para para la dinámica deberán ser públicos al momento de recuperar las publicaciones.

La aplicación sólo requerirá de un dato para recuperar los registros del muro grupal, éste es el ID compuesto de 10 dígitos que Facebook asigna a cada objeto que es creado, sea usuario, grupo, publicación, etc. En este caso el ID es proporcionado al sistema y enviado como parámetro para solicitar el objeto JSON (Java Script Object Notation) que contiene los usuarios $\mathrm{y}$ las publicaciones y comentarios realizados en el muro, instanciando la función predefinida en el SDK de Faceboook FacebookRequest, previamente se realiza unas configuraciones dentro del Facebook para que la URL de la aplicación sea reconocida como aplicación de Facebook, quedando el requerimiento de la siguiente forma:

\section{Srequestfeed=new}

FacebookRequest(\$session,'GET','/'.\$_SESSION['gidd'].'/feed?fields=message, from,comments');

\section{B. El Modelo de Requerimientos}

La especificación de requisitos, en UWE se realiza mediante el modelo de requerimientos, el cual considera el modelado de casos de uso de UML; donde con actor se ha modelado al usuario que en éste caso sería una instancia de un usuario de Facebook, ya que requerirá haber iniciado de sesión en Facebook para hacer uso de la aplicación, en el diagrama de manera genérica se ha nombrado Analizador, pudiendo ser el profesor, alumno, tutor, etc.., los casos de uso modelados es decir las funcionalidades que proporciona la aplicación son la recuperación de datos registrados en el muro grupal (usuarios, publicación, fecha de publicación), almacenar los datos en una base de datos de la aplicación, con los indicadores realizar un análisis cada grupo y entre grupos y una comparación entre grupos en base a los índices grupales y por último la generación de gráficas. La Figura 3 ilustra el diagrama de casos de uso para la aplicación Web.

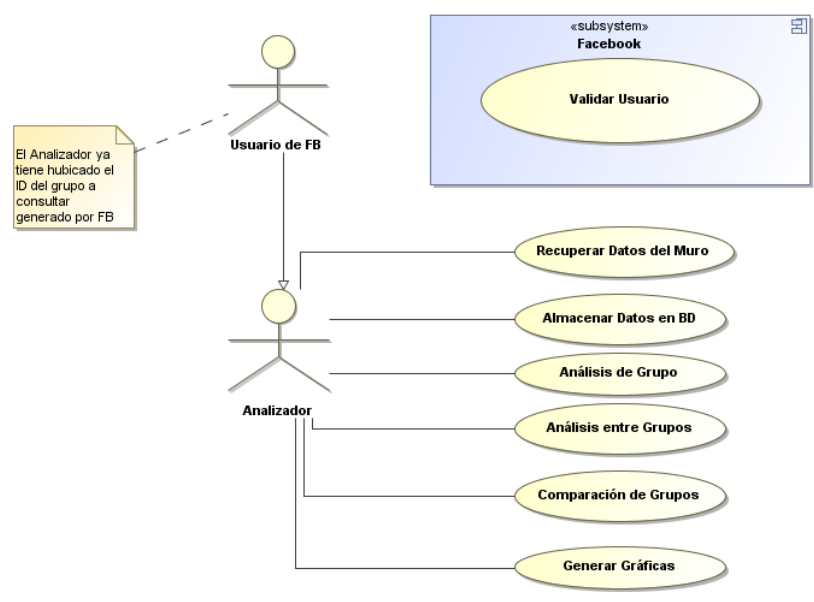

Fig. 3. Casos de Uso para la HAI.

El funcionamiento de la herramienta depende en gran medida de la estrategia del uso de OA en las publicaciones dentro del muro grupal. Y por ende la comunicación de una actividad complementada con alguna técnica colaborativa que promueva interacción en la tarea a realizar. Es de recalcar que el usuario de la aplicación, por ejemplo el Profesor no necesariamente tendría que ser el administrador del grupo o ser miembro del grupo debido a que el grupo se crea para llevar a cabo la tarea asignada, y al momento de obtener datos con la aplicación se configura como público, por lo que el grupo fue creado con la finalidad educativa de la obtención del nivel de colaboración, por lo que si este no se encuentra configurado como público simplemente no se podrá obtener las publicaciones del muro, por lo que una vez obtenido los datos puede cambiarse la configuración a grupo cerrado e inclusive eliminar el grupo por completo.

Los resultados de grupo se visualizan en una tabla con índices de cada uno de los cuatro atributos en donde la primera columna corresponde a los nombres de los integrantes como usuarios de Facebook.

El tipo de gráfico utilizado para representar los indicadores generados, es el de Gráfico de Barras, debido a la facilidad de interpretar a simple vista la diferencia obtenida en los indicadores.

\section{El Modelo del Dominio}

El modelo conceptual para el contenido (Modelo del Dominio) proporciona una especificación visual de la información en el dominio relevante de la aplicación Web, mostrando los principales elementos que integran la aplicación Web para su desarrollo y funcionamiento, con los diagramas de clases de UML, siendo éstos: la clase "Presentación Dinámica", para adaptar la interfaz del sitio web al tamaño del dispositivo en que se visualice, desde una PC a una Tablet $\mathrm{u}$ otro dispositivo, en conjunto las clase "etiquetas", "Menú", "Enlaces", "Barras", "Tablas Dinámicas de Resultados", "Gráficas", "botón" e "Imagen". En la Figura 4 se presenta el diagrama de clases para el modelo de contenido.

\section{El Modelo de Navegación}

La navegación de cada nodo y link es decir las páginas y enlaces que van de una página a otra, se muestra en el siguiente diagrama de navegación (Ver Fig. 5). 


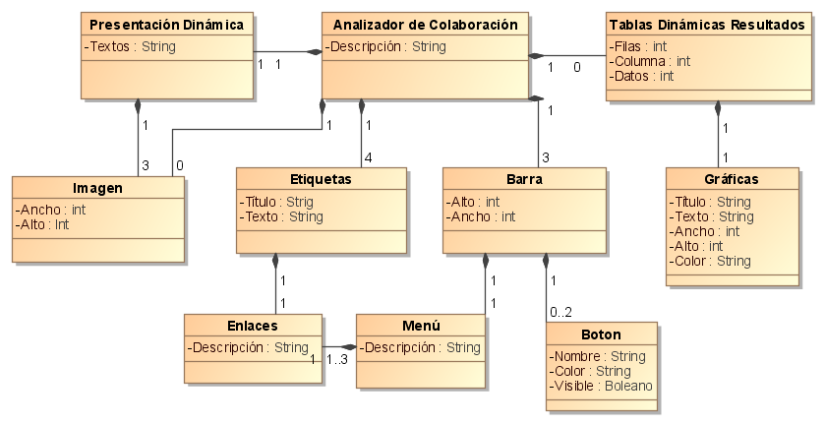

Fig. 4. Modelo de Contenido.

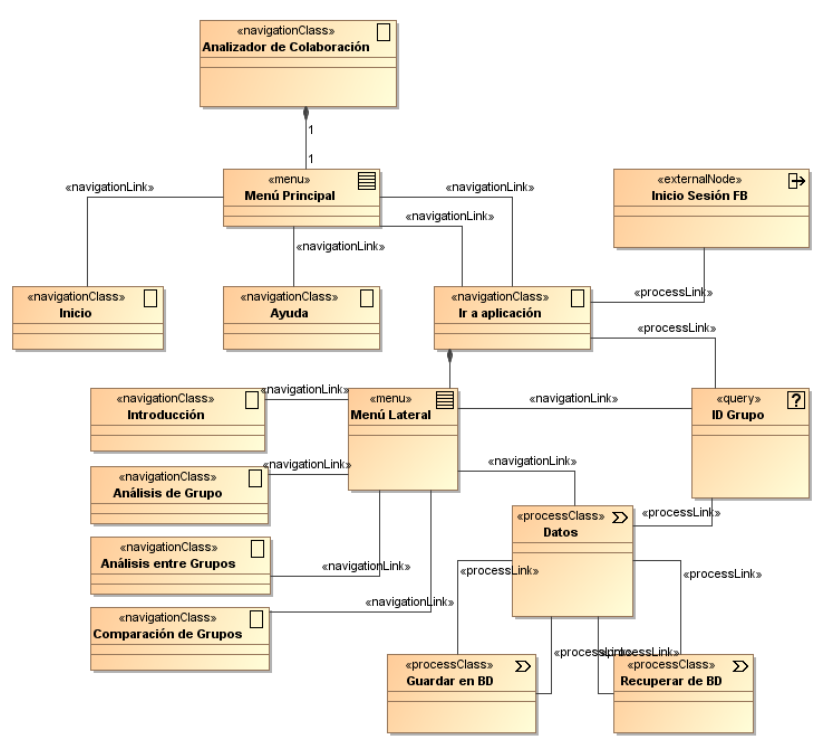

Fig. 5. Modelo de Navegación.

En el "Menú Principal" se ha anclado direccionar a la página principal, ésta misma nos da acceso a la clase "Ir a aplicación", realizar un "inicio de Sesión en FB" lo cual se ha anclado y representado con el extereotipo $<<$ externalNode $>>$, el funcionamiento de la recuperación del objeto JSON (Java Script Object Notation) requiere del $<<$ query $>>$ "ID Grupo", obtenido los datos se pueden "Guardar en BD", "Recuperar en BD” para luego poder realizar los análisis correspondientes a través del "Menú Lateral".

\section{E. El Modelo de Presentación}

El modelo de presentación, una visión abstracta de la interfaz de usuario (IU), con los elementos básicos que integrará la IU de la página principal "Analizador de Colaboración", el cual facilitó el diseño de los elementos en cuanto a su posición y funcionamiento es mostrado en la Figura 6.

Como se observa en la figura anterior fue modelada por «presentationPage», las clases «text», y «anchor». En la Figura 7 se modela la página de aplicación "paginaAplicación" donde se solicita el ID de grupo para recuperar las publicaciones del muro grupal que pertenecen a dicho grupo, lo elementos empleados en este modelo son para introducir el ID de grupo utilizado «textiput»y un «button» para el botón de envío, así como «button» de inicio o para cerrar la sesión iniciada de FB.

Para la tabla de datos (Figura 8) se empleó «presentatioAlternatives» el cual tiene un filtro de búsqueda de los datos así como «button». Finalmente, la Figura 9 es la última que corresponde al modelado del diseño de las vistas y tiene que ver en la presentación de los elementos para los datos calculados.

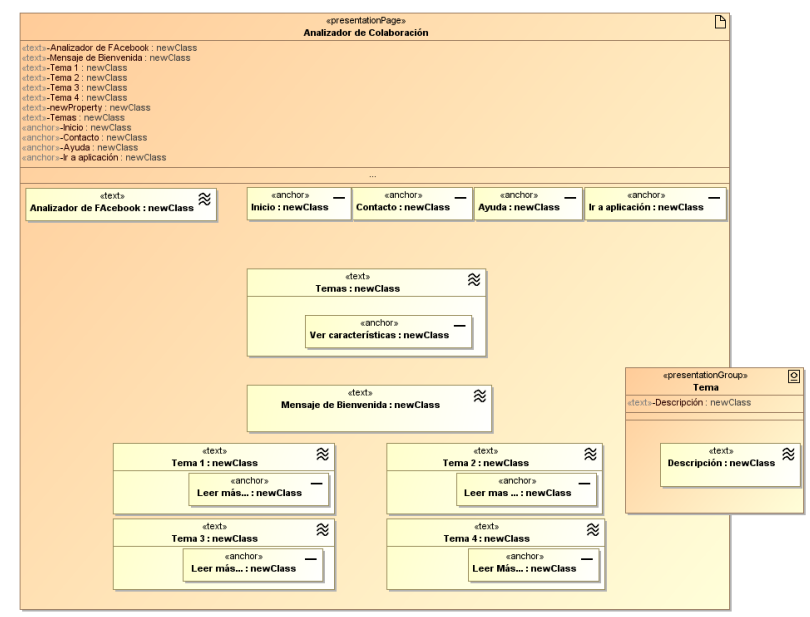

Fig. 6. Página de presentación: Analizador de Colaboración.

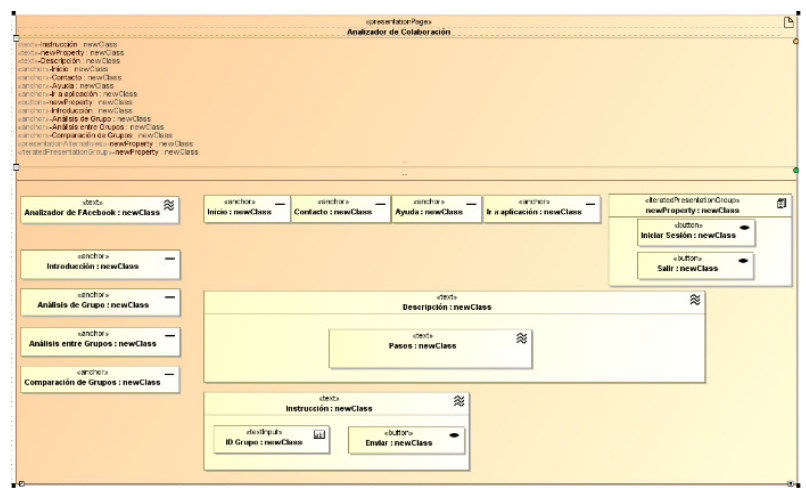

Fig. 7. Página de presentación: Analizador de Colaboración.

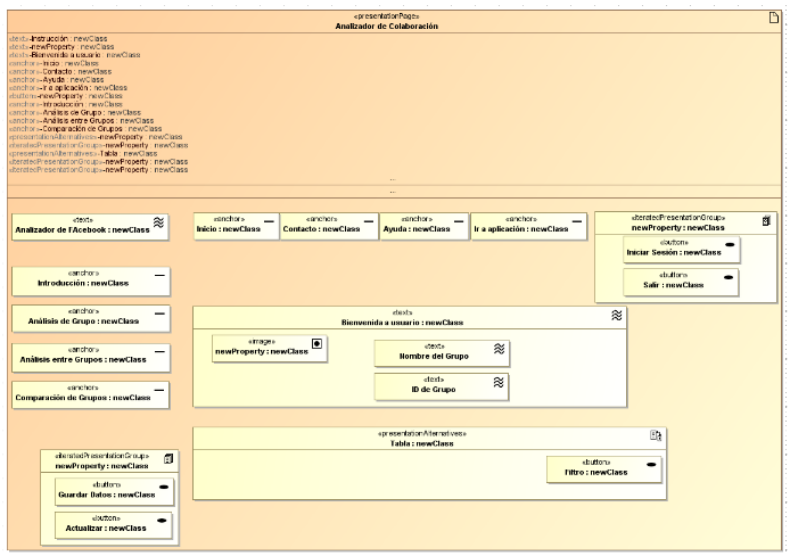

Fig. 8. Página de presentación: Resultados.

Los elementos que la imagen integra (Figura 9) es la selección del grupo al cual se le aplicará los indicadores programados y la gráfica correspondiente a los mismos, modelado en una clase «presentatioAlternatives» ya que variará la selección de acuerdo a una selección de un solo grupo, selección de varios grupos, al igual las tablas corresponderá a la selección del menú lateral, de igual forma indicadores aplicados a un solo grupo, a varios al mismo tiempo o a realizar una comparación de grupos, por lo que el modelado de la página para el análisis y comparación de grupos será la misma.

\section{F. El Modelo de Proceso}

El modelo del proceso (las acciones de las clases de proceso) se encuentra compuesto de dos tipos de modelos: El Modelo de estructura del proceso, el cual que describe las relaciones entre las diferentes clases de proceso, y Modelo de 
flujo del proceso, que específica las actividades conectadas con cada «processClass».

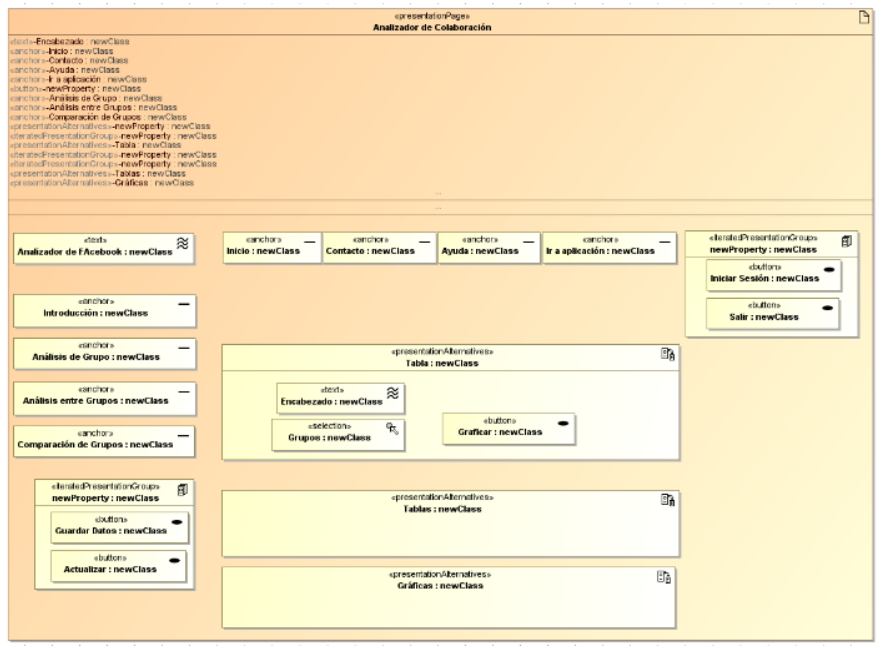

Fig. 9. Página de presentación: Datos para el análisis.

La Figura 10 presenta un diagrama de clases donde se describen las relaciones entre las diferentes clases de proceso (Modelo de estructura del proceso).

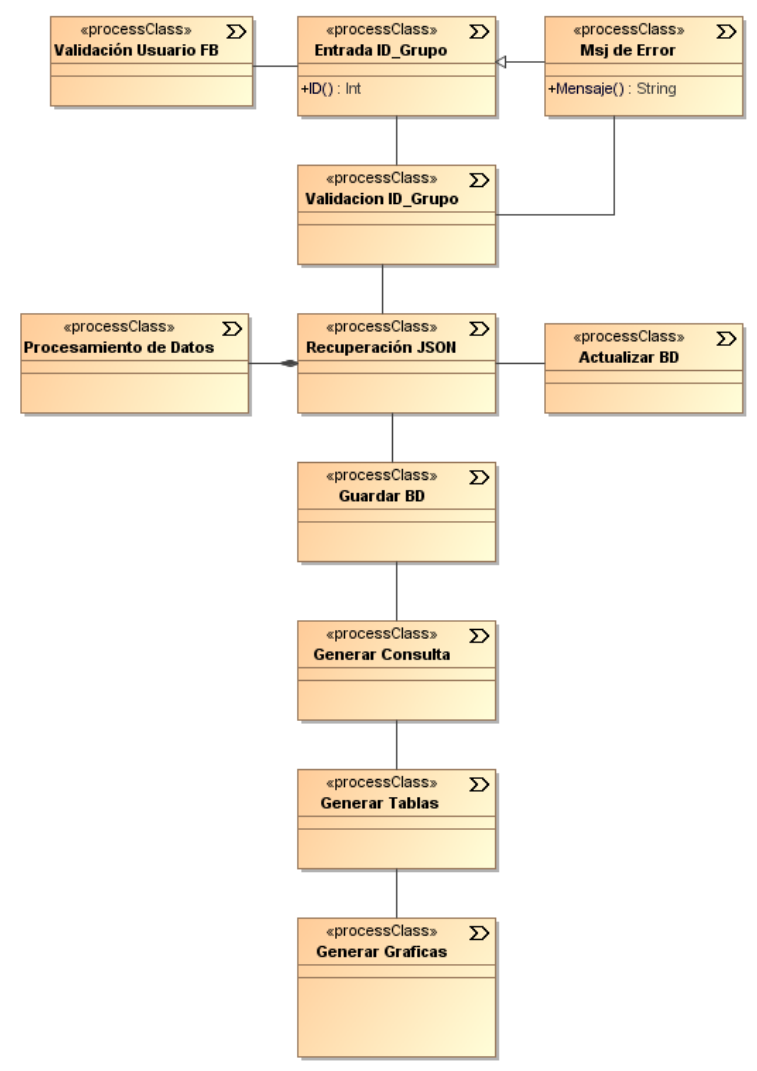

Fig. 10. Estructura de proceso.

En relación con el flujo del proceso, la Figura 11 ilustra el diagrama que especifica las actividades conectadas al «processClass», "Iniciar Aplicación"; dicho diagrama muestra que para iniciar la aplicación el usuario proporcionará al sistema el ID de Grupo generado por Facebook, el sistema validará tiene inicio de sesión.

En la Figura 12 se ilustra el diagrama de actividad para el proceso "Buscar ID". Al activar el botón, donde sólo si es correcta y existe la información realiza la petición a través de una página que contiene funciones del sdk de php, y un ciclo para obtener en un solo objeto los datos requeridos, después de que el objeto está completo es devuelto en un objeto JSON
(Java Script Object Notation) y la página que recibe dicho objeto descompone el objeto y filtra los datos para presentarlos en una tabla, en caso contrario permanece en la página para intentar una nueva consulta.

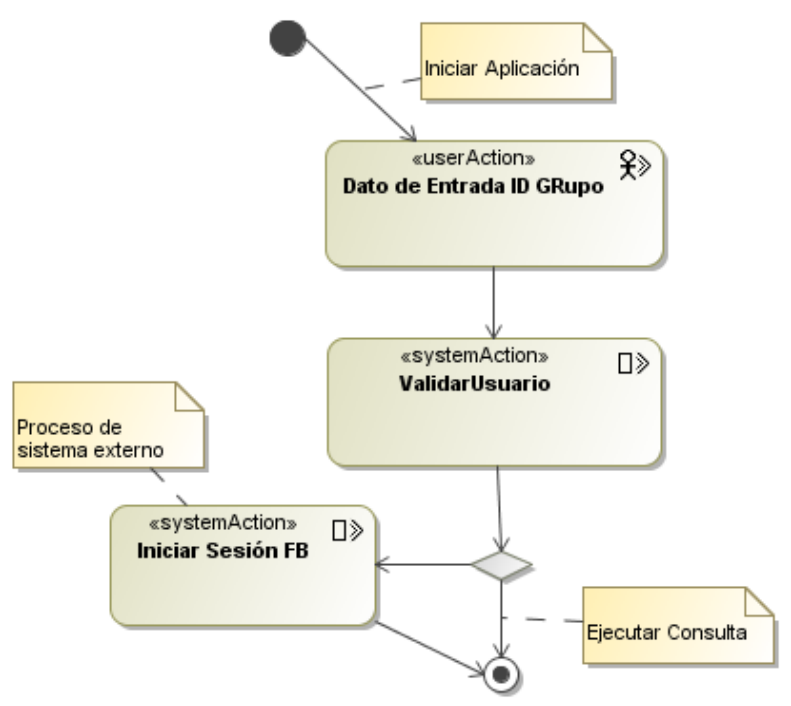

Fig. 11. Flujo de proceso: Iniciar Aplicación.

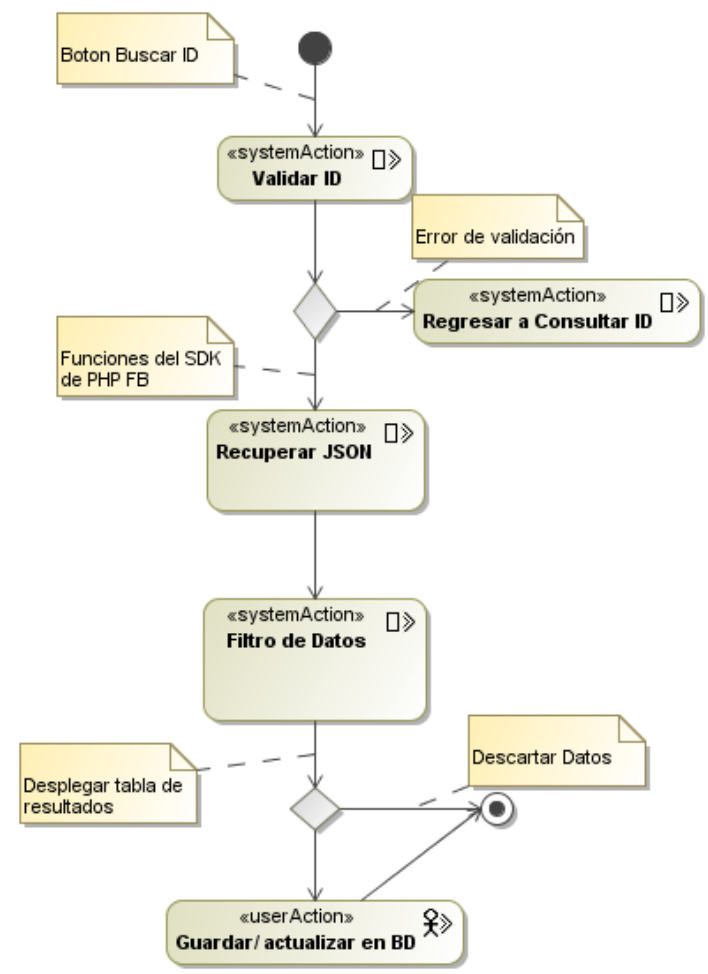

Fig. 12. Flujo de proceso: Buscar ID.

En cuanto al diagrama de actividad para el proceso "Análisis de grupo" (ver Figura 13), en esta se muestra que el usuario debe seleccionar el grupo del que desea tener los indicadores de colaboración de entre una lista de los grupos que el sistema pone a disposición y que han sido previamente guardados para luego aplicar el modelo de colaboración, y en base a los resultados de la tabla de resultados genera las gráficas de barras correspondientes a cada uno de los tres grupos de indicadores (habilidades colaborativas, participación $\mathrm{y}$ uso de las habilidades colaborativas), de forma individual y grupal, obtenidos los resultados el usuario puede almacenar en la base de datos los resultados obtenidos de los grupos, esto con el fin de realizar un análisis de grupos como se muestra en el último diagrama. 


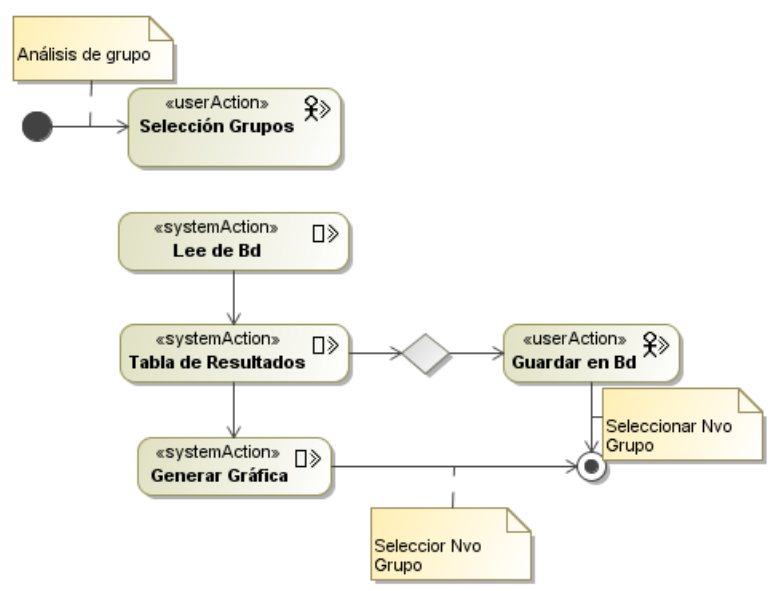

Fig. 13. Flujo de proceso: Análisis de grupo.

Un último proceso "Análisis entre grupos", para realizar una comparación entre grupos, donde según el diagrama el usuario debe seleccionar en este caso los grupos a comparar de entre una lista que el sistema pone a disposición, de los resultados grupales se generan gráficas de barras correspondientes a cada uno de los tres grupos de indicadores (habilidades colaborativas, participación y uso de las habilidades colaborativas) y de manera similar funciona el proceso "Mejor grupo", el usuario hace una selección de más de un grupo ,sistema toma sólo los datos globales, es decir la habilidad colaborativa que corresponde al grupo, la participación total del grupo, desplegando de nuevo una tabla de resultados y las gráficas de barras correspondiente.

\section{Prestaciones de la Herramienta PARA El ANÁlisis de LA INTERACCIÓN (HAI).}

En esta sección se presenta algunas funcionalidades de la Herramienta para el Análisis de la Interacción desarrollada con el diseño presentado en la sección anterior, se muestra la secuencia de acciones a realizar para la obtención de los indicadores de colaboración. La página de Inicio se ilustra en la Figura 14; en la parte superior derecha se puede observar un menú con la opción para ejecutar la aplicación.

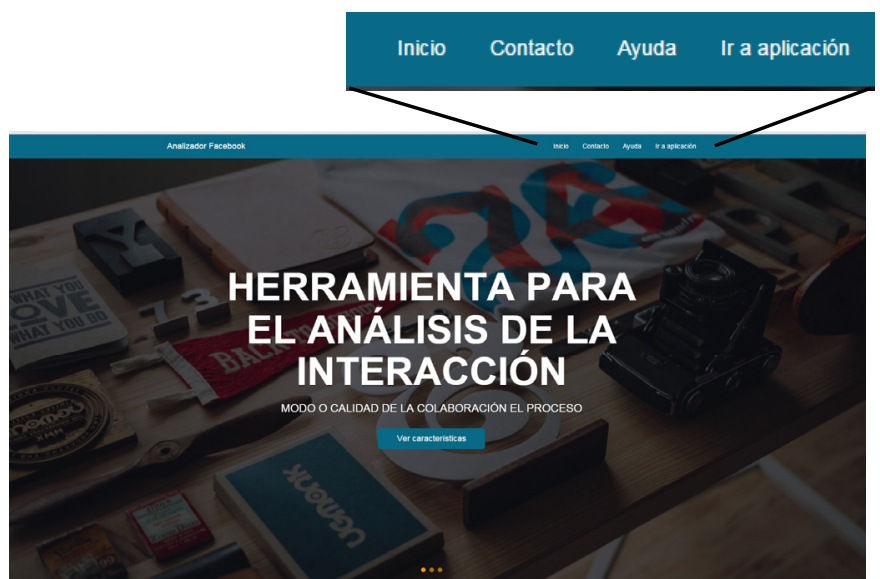

Figura 14. Vista de la Página de Inicio.

Debido a que la HAI puede ser utilizada por un profesor con bajo nivel de expertiz en el uso de las TIC, la interacción se ha diseñado de tal manera que guía paso a paso al usuario en la ejecución se sus funcionalidades; la Figura 15 ilustra los tres pasos previos para la obtención de indicadores.

Como primer paso se le pide al usuarios haber iniciado sesión en Facebook; en el paso número dos se indica que para la obtención de los datos del muro se requiere que éste sea el administrador del grupo, aunque bastaría en configurar como público el grupo para poder recuperar los datos; finalmente se solicita el Identificador del grupo (ID); dicho Identificador es un número de quince dígitos generado y asignado por Facebook al momento de crear un grupo, por lo que se puede obtener del URL al momento de entrar al grupo (Ver Figura 16); en la figura se puede observar que el ID del grupo seleccionado es: 777672448945294.

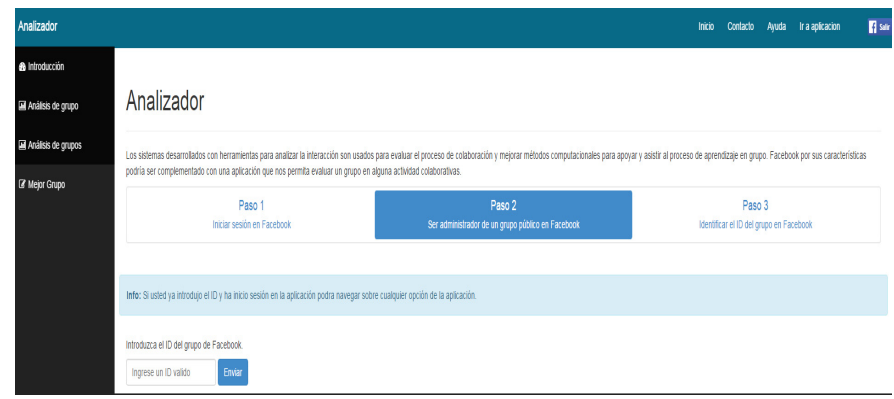

Fig. 15. Vista de la aplicación.

https://www.facebook.com/groups/777672448945294/

Fig. 16. URL creada para el grupo.

La aplicación validará que haya una sesión iniciada, de lo contrario redireccionará el control a un inicio de sesión directamente en Facebook (Figura 17).

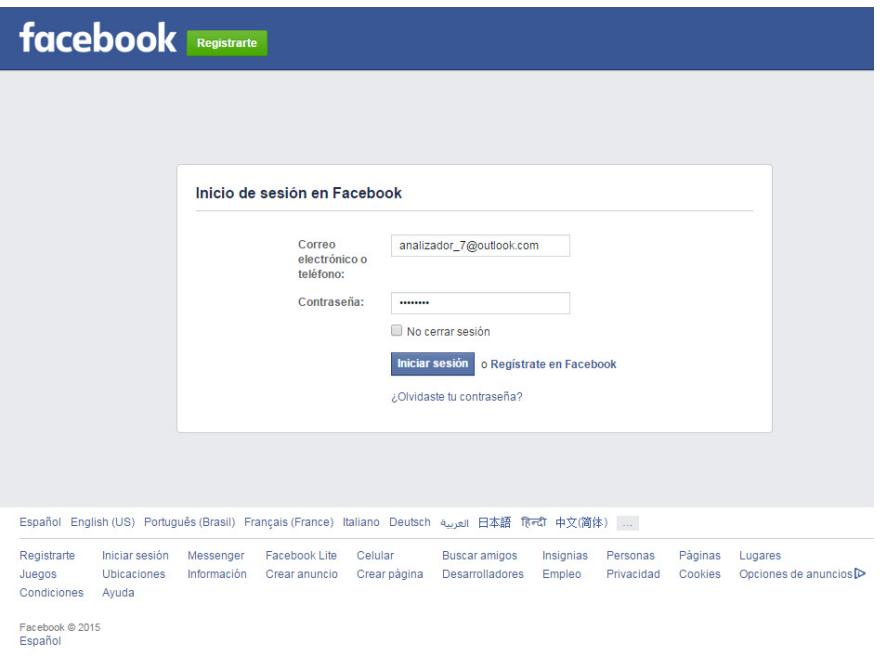

Fig. 17. Inicio de sesión en Facebook.

Finalmente, una vez que el ID es introducido por el usuario, la aplicación recupera las publicaciones registradas en el muro, y el usuario puede guardarlas en la base de datos local o actualizarlas si los datos ya han sido previamente guardados. Después obtener y guardar los datos en la base de datos, se podrán generar y obtener los indicadores en el menú ubicado en la sección izquierda del HAI.

Si lo que se quiere es obtener los niveles de colaboración de los integrantes de un grupo, es decir, obtener los indicadores individuales, se deberá seleccionar "Análisis de grupo"; la página hará visible los datos guardados en la base de datos para que se pueda seleccionar el grupo de cual se desena analizar los indicadores individuales. Una vez seleccionado el grupo, se visualizará detalles de los de los integrantes, así como un conjunto de gráficos de barras vinculados con los indicadores generados. La Figura 18 ilustra una consulta realizada al HAI 
luego de las sesiones experimentales con un conjunto de grupos de estudiantes.

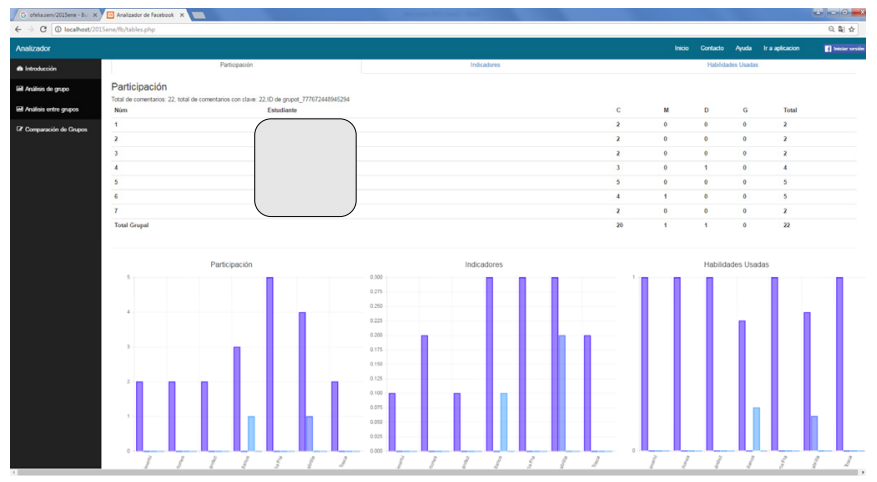

Fig. 18. Indicadores Individuales en el HAI

(Con nombres de estudiantes ocultos).

Los indicadores generados para cada integrante del grupo seleccionado, corresponden a: (1) en la primera pestaña, a la participación de cada estudiante (tal y como se ilustra en la Figura 18), (2) en la segunda pestaña a los indicadores de cada uno de los atributos de la colaboración (comunicación, mantenimiento de la confianza, dirección y gestión de conflictos), (3) y la última pestaña corresponde a cómo cada una de las habilidades colaborativas fueron utilizadas por los estudiantes en una sesión de trabajo. Independientemente de la pestaña seleccionada, la HAI despliega los tres conjuntos de gráficos de barras vinculados con los indicadores individuales.

En el caso de los indicadores grupales, se debe hacer la selección del menú "Análisis grupal”; la aplicación permitirá seleccionar uno o varios grupos, para los cuales se podrá visualizar tres pestañas similares a las desplegadas en el análisis individual, pero en este caso, la HAI de mostrarán valores y gráficos correspondientes a los indicadores grupales (ver Figura 19) generados con base en el mecanismo de modelado descrito en la sección tres.

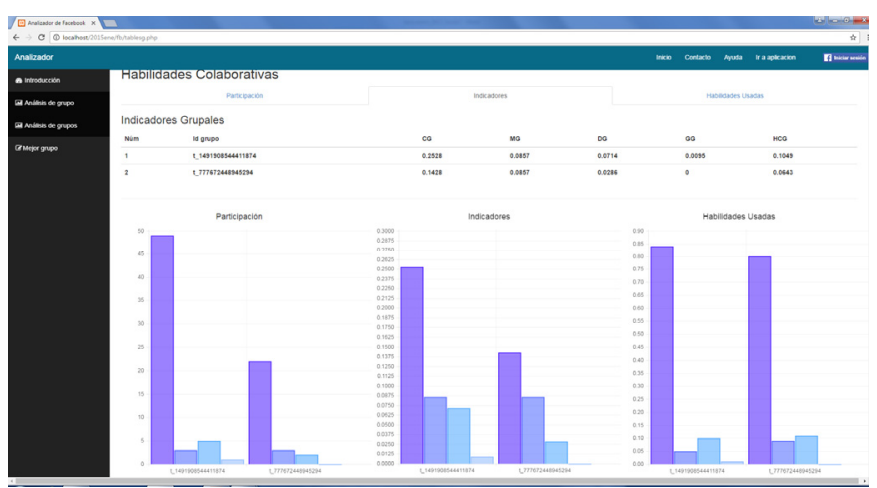

Fig. 19. Indicadores Grupales en el HAI.

\section{CONCLUSIONES}

En este trabajo se ha presentado la aplicación de Ingeniería Web para el desarrollo de una aplicación utilizando como metodología UWE, la cual utiliza UML a través de un conjunto de modelos que permiten especificar de manera clara y sencilla la funcionalidad, características y elementos que conforma las especificaciones de la aplicación Web. La aplicación descrita en este artículo, realiza una conexión con la red social Facebook y tiene como finalidad apoyar el análisis de la colaboración; la aplicación ha sido desarrollada como parte de un proyecto de investigación que tiene como objetivo realizar un análisis de la colaboración en escenarios de aprendizaje asistidos por herramientas software de libre distribución. Para lo anterior, la herramienta automatiza un Modelo de Indicadores, con el cual es posible analizar gráficamente, el comportamiento registrado de los individuos y de los grupos, luego de un conjunto de sesiones de aprendizaje, en las cuales la interacción ha sido mediada a través de Facebook.

Al momento de redactar este trabajo los autores se encuentran analizando la información obtenida de la experimentación realizada con la HAI, donde además de validar la utilización de la red social Facebook, en el diseño de un escenario de aprendizaje colaborativo, se validan las prestaciones de la aplicación, con la intención de identificar aspectos que permitan una mejor interpretación de resultados.

\section{REFERENCIAS}

[1] C.A. Ellis, S.J. Gibbs, \& G. Rein: Groupware: some issues and experiences. ACM, 34(1), pp. 39-58, 1991.

[2] G. Stahl, T. Koschmann, \& D. Suthers: Computer supported collaborative learning: An historical perspective. In R. K. Sawyer (Ed.), Cambridge handbook of the learning sciences, pp. 409-426, Cambridge Univiversity Press, 2006.

[3] P. Dillenbourg, M. Baker, A. Blaye, \& C.O. Malley: The evolution of research on collaborative learning. In E. Spada \& P. Reiman (Eds) Learning in Humans and Machine: Towards an interdisciplinary learning science, pp. 189-211, Oxford: Elsevier, 1996

[4] C. Collazos, L. Guerrero, J. Pino, S. Renzi, J. Klobas, M. Ortega, M. Redondo, C. Bravo: Evaluating Collaborative Learning Processes using System-based Measurement. Educational Technology \& Society, pp. 257-274, 2007.

[5] J. Roschelle \& S. Teasley: The Construction of Shared Knowledge in Collaborative Problem Solving. In C.E. O'Malley (Ed.) Computer-Supported Collaborative Learning. pp. 69-197, Berlin: Springer-Verlag, 1991.

[6] S. Murugesan, Y. Deshpande, S. Hansen, A. Ginige: Web Engineering: A New Discipline for Development of Web-based Systems. Vol. 2016 of the series Lecture Notes in Computer Science, pp 3-13, 2001.

[7] S. Daniel, R. Gustavo: The object-oriented hypermedia design model. ACM, 38(8), 45-46, 1995.

[8] S. Ceri, P. Fraternali, A. Bongio: Web Modeling Language (WebML): a modeling language for designing Web sites. Computer Networks, 33(1-6), pp. 137-157, 2000.

[9] N. Koch, A. Knapp, G. Zhang, H. Baumeister: Uml-Based Web Engineering An Approach Based on Standards. In HumanComputers Interaction Series Springer, pp. 157-191, 2008.

[10] R. Aguilar: Entrenamiento de equipos : una estrategia asistida por entornos virtuales inteligentes. Tesis Doctoral de la Universidad Politécnica de Madrid, 2008.

[11] M. McManus, \& R. Aiken: Monitoring computer-based problem solving. Journal of Artificial Intelligence in Education, pp. 307336, 1995.

[12] D. Johnson, \& R. Johnson: Learning together and alone: cooperative, competitive and individualistic learning, 5th ed. Boston, 1999.

[13] A. Dimitracopoulou, P. Dillenbourg, \& U. Hoppe: Interaction analysis supporting participants during technology -based collaborative activities. In Workshop summary: CSCL SIG European Symposium, Lausanne, 2004.

[14] A. Soller, P. Jermann, M. Mühlenbrock \& A. Martínez: Designing computational models of collaborative learning interaction: 2nd International Workshop on Designing Computational Models of Collaborative Learning Interaction at ITS. Maceió, Brazil, 2004. 


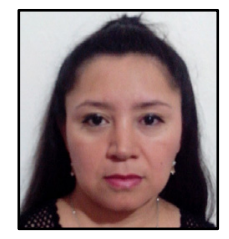

Ofelia Uicab Aldana es Ingeniera en Sistemas por la Universidad Latino. Maestra en Ciencias Computacionales por la Universidad Autónoma de Yucatán; su proyecto de Tesis fue desarrollado en el ámbito del Aprendizaje Colaborativo Asistido por Computadora. Sus áreas de interés son la Ingeniería de Software y la Informática Educativa.

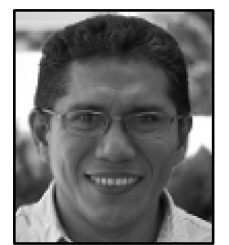

Juan Pablo Ucán Pech es Doctor en Sistemas Computacionales por la Unidad de Posgrado e Investigación de la Universidad del Sur, México. Maestro en Sistemas Computacionales con especialidad en Ingeniería de Software por el Instituto Tecnológico de Mérida, México. Licenciado en Ciencias de la Computación por la Facultad de Matemáticas de la Universidad Autónoma de Yucatán, México. Actualmente es Profesor Titular en la Facultad de Matemáticas e integrante del Grupo Académico de Tecnologías para la Formación en Ingeniería de Software de la UADY. Su trabajo de investigación se centra en temas relacionados con la Ingeniería de Software, Ingeniería Web e Informática Educativa.

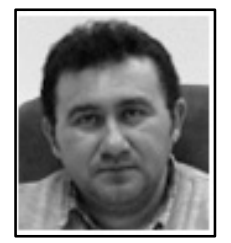

Raúl Antonio Aguilar Vera es Licenciado en Ciencias de la Computación por la Universidad Autónoma de Yucatán (UADY), Master en Ingeniería de Software y Doctor en el área de Informática por la Universidad Politécnica de Madrid, España. Actualmente es profesor de tiempo completo en la Facultad de Matemáticas de la UADY e integrante de Red Mexicana de Ingeniería de Software (REDMIS). Su trabajo de investigación incluye las siguientes áreas: Ingeniería de Software e Informática Educativa. 